\title{
Abrangência territorial de pesquisas com interface em Práticas Integrativas e Complementares em Saúde na Estratégia de Saúde da Família do Brasil
}

\section{Territorial scope of researches with an interface in Integrative and Complementary Health Practices in the Family Health Strategy in Brazil}

\author{
Alcance territorial de la investigación con \\ una interfaz en Prácticas Integradoras y \\ Complementarias en Salud en la Estrategia \\ de Salud Familiar en Brasil
}

\author{
Fernanda Elizabeth Sena Barbosa 1 \\ Maria Beatriz Lisboa Guimarães 2 \\ Carlos Renato dos Santos 3 \\ Adriana Falangola Benjamin Bezerra 2 \\ Charles Dalcanale Tesser 4 \\ Islandia Maria Carvalho de Sousa 1
}

doi: 10.1590/0102-311X00123420

Agradecemos os comentários de Barros et al. ${ }^{1}$ e apresentamos dados complementares ao artigo de Barbosa et al. 2 que não foram publicados no original, mas de interesse, na mesma direção do conteúdo abordado por Barros et al.

A Figura 1 representa a abrangência de cobertura das duas fontes de dados que subsidiaram a concepção do artigo. Dos 5.574 municípios brasileiros, o Programa Nacional de Melhoria do Acesso e da Qualidade da Atenção Básica (PMAQ) avaliou 5.041 e o Inquérito Nacional de Práticas Integrativas e Complementares em Saúde no SUS (Inquérito) 1.617. Foram identificados 1.487 municípios com participação nas duas pesquisas.

As distribuições geográficas do Inquérito e do PMAQ mostram que o cruzamento dos bancos permitiu o aumento da abrangência do estudo, com consequente inferência acerca da oferta de Práticas Integrativas e Complementares em Saúde (PICS) no país. Por ser um programa do Ministério da Saúde de avaliação da atenção básica, o PMAQ tinha a premissa de abranger todos os municípios brasileiros. Da mesma forma, o Inquérito também se propôs a atingir todos os municípios. Embora 5.520 municípios tenham recebido o convite para participar do Inquérito, supomos que aqueles que aceitaram tinham interesse, curiosidade ou demanda interna de profissionais acerca das PICS. Esses representam uma amostra considerável e satisfatória para alguma generalização com cautela, embora sujeita a vieses de seleção.

Um desses vieses pode ser que, sendo os gestores municipais maioria dentre os respondentes 749 (46,4\%) coordenadores da atenção primária e 605 (37,4\%) secretários de saúde -, provavelmente estes têm desejo de ofertar as PICS ou possuem oferta legitimada pela gestão ou tenha iniciativa de profissionais, a ponto de fazê-los reconhecer o tema e se dispor a participar. Se isso for verdade, a inferência é realizada no sentido do que os dados indicam, ou seja, de que a maioria dos municípios respondentes não tem oferta estruturada de PICS, o que contradiz os dados oficiais do Ministério da Saúde sobre a oferta de PICS 3.

Para interpretar tal contradição, devemos lembrar que a magnitude da oferta de PICS, de acordo com os dados do Ministério da Saúde, é proveniente do Cadastro Nacional de Estabelecimentos de Saúde (CNES), do Sistema de Informação Ambulatorial (SIA) e do Sistema de Informação em Saúde para Atenção Básica (SISAB). No CNES pode haver sub-registro ou sobrerregistro, por não haver consequências ou auditorias a respeito do dado informado. No SIA e SISAB, um profissional, ao registrar
1 Instituto Aggeu Magalhães, Fundação Oswaldo Cruz, Recife, Brasil.

2 Departamento de Medicina Social, Universidade Federal de Pernambuco, Recife, Brasil.

3 Centro Acadêmico de Vitória, Universidade Federal de Pernambuco, Vitória de Santo Antão, Brasil. ${ }^{4}$ Departamento de Saúde Pública, Universidade Federal de Santa Catarina, Florianópolis, Brasil.

\section{Correspondência}

I. M. C. Sousa

Departamento de Saúde

Coletiva, Instituto Aggeu

Magalhães, Fundação

Oswaldo Cruz.

Av. Prof. Moraes Rego $s / n$, Campus da UFPE, Recife, PE 50670420, Brasil.

islandia@cpqam.fiocruz.br 
Municípios com participação no Inquérito Nacional de Práticas Integrativas e Complementares em Saúde no SUS (Inquérito) e no Programa Nacional de Melhoria do Acesso e da Qualidade da Atenção Básica (PMAQ).

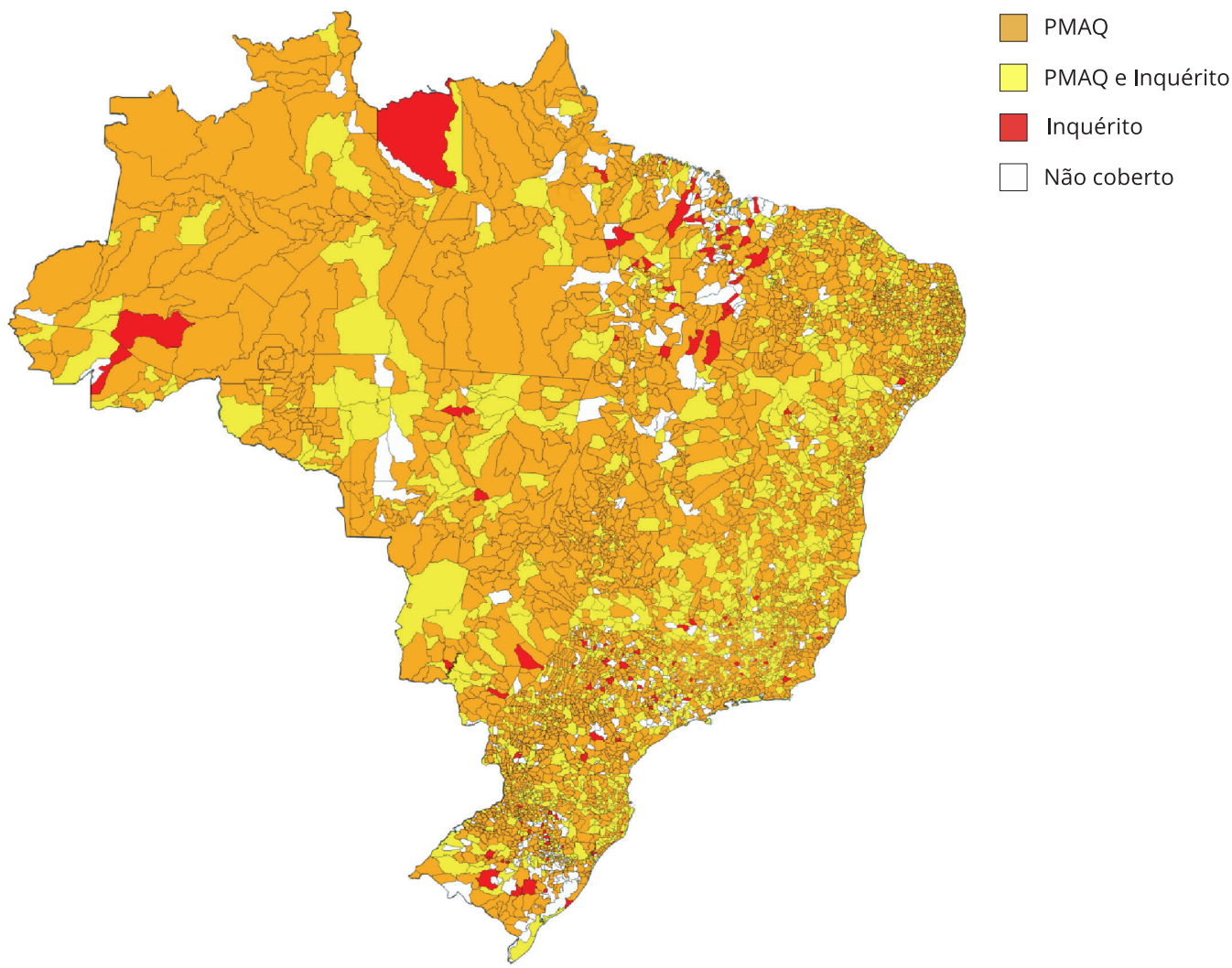

uma atividade de PICS, converte seu município em ofertante nas estatísticas governamentais 4 , porém esta oferta pode ser ocasional e pontual, não representando uma oferta sistemática e institucionalmente apoiada. Os dados do Inquérito e do PMAQ reforçam a nossa intepretação e de Barros et al. 1 de que o protagonismo da oferta está concentrado nos profissionais, sendo pequena a incorporação da mesma pelos gestores.

O Inquérito e o estudo referenciados por Barros et al. 1, realizados em São Paulo, sugerem que a análise sobre a oferta de PICS no território nacional requer maior aprofundamento. Há interesse de parcela dos profissionais de saúde, cuja magnitude é difícil de estimar com base nos estudos aqui mencionados e nas fontes oficiais, em ofertar um cuidado com abordagem integral e PICS. Contudo, as motivações e o modo como esse cuidado é ofertado devem ser explorados inclusive para avaliar a segurança, qualidade e efetividade.

No Inquérito não foi detalhado o perfil do gestor e, portanto, não permite inferência sobre que fatores e experiências podem tê-lo influenciado quanto à oferta ou não de PICS no seu município. Outros estudos que estamos conduzindo abordam esses fatores e poderão elucidá-los. Parece-nos ser importante, também, identificar o que mobiliza os profissionais a desempenharem funções para as quais não foram efetivamente contratados. 


\section{Colaboradores}

F. E. S. Barbosa contribuiu com a concepção, redação e análise em todas as etapas. M. B. L. Guimarães e C. R. Santos contribuíram com a análise dos dados e revisão. A. F. B. Bezerra e C. D. Tesser revisaram o texto. I. M. C. Sousa contribuiu com a redação e análise dos dados e revisão.

\section{Informações adicionais}

ORCID: Fernanda Elizabeth Sena Barbosa (00000003-4336-8174); Maria Beatriz Lisboa Guimarães (0000-0001-8554-600X); Carlos Renato dos Santos (0000-0001-8367-7006); Adriana Falangola Benjamin Bezerra (0000-0002-5278-3727); Charles Dalcanale Tesser (0000-0003-0650-8289); Islandia Maria Carvalho de Sousa (0000-0001-9324-4896).
1. Barros NF, Francisco PMSB, Sousa LA. Carta às Editoras sobre o artigo de Barbosa et al. Cad Saúde Pública 2020; 36:e0062320.

2. Barbosa FES, Guimarães MBL, Santos CR, Bezerra AFB, Tesser CD, Sousa IMC. Oferta de Práticas Integrativas e Complementares em Saúde na Estratégia Saúde da Família no Brasil. Cad Saúde Pública 2020; 36:e00208818.

3. Secretaria de Atenção Primária, Ministério da Saúde. Práticas Integrativas e Complementares. Onde tem Práticas Integrativas e Complementares (PICS)? https://saude.gov.br/saudede-a-z/praticas-integrativas-e-complementa res (acessado em 28/Abr/2020).

4. Tesser CD, Sousa IMC, Nascimento MC. Práticas Integrativas e Complementares na Atenção Primária à Saúde brasileira. Saúde Debate 2018; 42 (spe 1):174-88.

Recebido em 13/Mai/2020

Aprovado em 04/Jun/2020 